\title{
Generated Sets of the Complete Semigroup Binary Relations Defined by Semilattices of the Class $\Sigma_{8}(X, n+k+1)$
}

\author{
Yasha Diasamidze, Omari Givradze, Nino Tsinaridze, Giuli Tavdgiridze \\ Faculty of Mathematics, Physics and Computer Sciences, Shota Rustaveli State University, Batumi, Georgia \\ Email: n.tsinaridze@bsu.edu.ge
}

How to cite this paper: Diasamidze, Y., Givradze, O., Tsinaridze, N. and Tavdgiridze, G. (2018) Generated Sets of the Complete Semigroup Binary Relations Defined by Semilattices of the Class $\Sigma_{8}(X, n+k+1)$. Applied Mathematics, 9, 369-382.

https://doi.org/10.4236/am.2018.94028

Received: March 22, 2018

Accepted: April 24, 2018

Published: April 27, 2018

Copyright $\odot 2018$ by authors and Scientific Research Publishing Inc. This work is licensed under the Creative Commons Attribution International License (CC BY 4.0).

http://creativecommons.org/licenses/by/4.0/

\begin{abstract}
In this article, we study generated sets of the complete semigroups of binary relations defined by $X$-semilattices unions of the class $\Sigma_{8}(X, n+k+1)$, and find uniquely irreducible generating set for the given semigroups.
\end{abstract}

\section{Keywords}

Semigroup, Semilattice, Binary Relation

\section{Introduction}

Let $X$ be an arbitrary nonempty set, $D$ is an $X$-semilattice of unions which is closed with respect to the set-theoretic union of elements from $D, f$ be an arbitrary mapping of the set $X$ in the set $D$. To each mapping $f$ we put into correspondence a binary relation $\alpha_{f}$ on the set $X$ that satisfies the condition $\alpha_{f}=\bigcup_{x \in X}(\{x\} \times f(x))$. The set of all such $\alpha_{f}(f: X \rightarrow D)$ is denoted by $B_{X}(D)$. It is easy to prove that $B_{X}(D)$ is a semigroup with respect to the operation of multiplication of binary relations, which is called a complete semigroup of binary relations defined by an $X$-semilattice of unions $D$.

We denote by $\varnothing$ an empty binary relation or an empty subset of the set $X$. The condition $(x, y) \in \alpha$ will be written in the form $x \alpha y$. Further, let $x, y \in X$, $Y \subseteq X, \alpha \in B_{X}(D), \breve{D}=\bigcup_{Y \in D} Y$ and $T \in D$. We denote by the symbols $y \alpha, Y \alpha, V(D, \alpha), X^{*}$ and $V\left(X^{*}, \alpha\right)$ the following sets:

$$
\begin{aligned}
& y \alpha=\{x \in X \mid y \alpha x\}, Y \alpha=\bigcup_{y \in Y} y \alpha, V(D, \alpha)=\{Y \alpha \mid Y \in D\}, \\
& X^{*}=\{Y \mid \varnothing \neq Y \subseteq X\}, V\left(X^{*}, \alpha\right)=\{Y \alpha \mid \varnothing \neq Y \subseteq X\},
\end{aligned}
$$




$$
D_{T}=\{Z \in D \mid T \subseteq Z\}, Y_{T}^{\alpha}=\{y \in X \mid y \alpha=T\} .
$$

It is well known the following statements:

Theorem 1.1. Let $D=\left\{\breve{D}, Z_{1}, Z_{2}, \cdots, Z_{m-1}\right\}$ be some finite $X$-semilattice of unions and $C(D)=\left\{P_{0}, P_{1}, P_{2}, \cdots, P_{m-1}\right\}$ be the family of sets of pairwise nonintersecting subsets of the set $X$ (the set $\varnothing$ can be repeated several times). If $\varphi$ is a mapping of the semilattice $D$ on the family of sets $C(D)$ which satisfies the conditions

$$
\varphi=\left(\begin{array}{ccccc}
\breve{D} & Z_{1} & Z_{2} & \cdots & Z_{m-1} \\
P_{0} & P_{1} & P_{2} & \cdots & P_{m-1}
\end{array}\right)
$$

and $\hat{D}_{Z}=D \backslash D_{Z}$, then the following equalities are valid:

$$
\breve{D}=P_{0} \cup P_{1} \cup P_{2} \cup \cdots \cup P_{m-1}, Z_{i}=P_{0} \cup \bigcup_{T \in \hat{D}_{Z_{i}}} \varphi(T) .
$$

In the sequel these equalities will be called formal.

It is proved that if the elements of the semilattice $D$ are represented in the form (1.1), then among the parameters $P_{i} \quad(0<i \leq m-1)$ there exist such parameters that cannot be empty sets for $D$. Such sets $P_{i}$ are called bases sources, where sets $P_{j} \quad(0 \leq j \leq m-1)$, which can be empty sets too are called completeness sources.

It is proved that under the mapping $\varphi$ the number of covering elements of the pre-image of a

bases source is always equal to one, while under the mapping $\varphi$ the number of covering elements of the pre-image of a completeness source either does not exist or is always greater than one (see [1] [2] chapter 11).

Definition 1.1. We say that an element $\alpha$ of the semigroup $B_{X}(D)$ is external if $\alpha \neq \delta \circ \beta$ for all $\delta, \beta \in B_{X}(D) \backslash\{\alpha\} \quad$ (see [1] [2] Definition 1.15.1).

It is well known, that if $B$ is all external elements of the semigroup $B_{X}(D)$ and $B^{\prime}$ is any generated set for the $B_{X}(D)$, then $B \subseteq B^{\prime}$ (see [1] [2] Lemma 1.15.1).

Definition 1.2. The representation $\alpha=\bigcup_{T \in D}\left(Y_{T}^{\alpha} \times T\right)$ of binary relation $\alpha$ is called quasinormal, if $\bigcup_{T \in D} Y_{T}^{\alpha}=X$ and $Y_{T}^{\alpha} \cap Y_{T^{\prime}}^{\alpha}=\varnothing$ for any $T, T^{\prime} \in D$, $T \neq T^{\prime} \quad$ (see [1] [2] chapter 1.11).

Definition 1.3. Let $\alpha, \beta \subseteq X \times X$. Their product $\delta=\alpha \circ \beta$ is defined as follows: $x \delta y \quad(x, y \in X)$ if there exists an element $z \in X$ such that $x \alpha z \beta y$ (see [1], chapter 1.3 ).

\section{Result}

Let $\Sigma_{8}(X, n+k+1) \quad(3 \leq k \leq n)$ be a class of all $X$-semilattices of unions whose every element is isomorphic to an $X$-semilattice of unions $D=\left\{Z_{1}, Z_{2}, \cdots, Z_{n+k}, \breve{D}\right\}$, which satisfies the condition:

$$
\begin{aligned}
& Z_{n+i} \subset Z_{i} \subset \breve{D},(i=1,2, \cdots, k) ; Z_{j} \subset \breve{D},(j=1,2, \cdots, n+k) ; \quad \text { (see Figure 1). } \\
& Z_{p} \backslash Z_{q} \neq \varnothing \text { and } Z_{q} \backslash Z_{p} \neq \varnothing(1 \leq p \neq q \leq n+k) .
\end{aligned}
$$




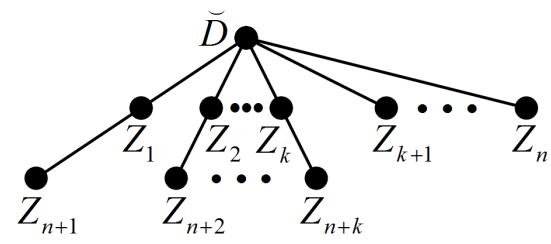

Figure 1. Diagram of the semilattice D.

It is easy to see that $\tilde{D}=\left\{Z_{1}, Z_{2}, \cdots, Z_{n+k}\right\}$ is irreducible generating set of the semilattice $D$.

Let $C(D)=\left\{P_{0}, P_{1}, P_{2}, \cdots, P_{n+k}\right\}$ be a family of sets, where $P_{0}, P_{1}, P_{2}, \cdots, P_{n+k}$ are pairwise disjoint subsets of the set $X$ and $\varphi=\left(\begin{array}{ccccc}\breve{D} & Z_{1} & Z_{2} & \cdots & Z_{n+k} \\ P_{0} & P_{1} & P_{2} & \cdots & P_{n+k}\end{array}\right)$ is a map ping of the semilattice $D$ onto the family of sets $C(D)$. Then the formal equalities of the semilattice $D$ have a form:

$$
\breve{D}=\bigcup_{i=0}^{n+k} P_{i} ; \quad Z_{j}=\bigcup_{\substack{i=0, i \neq j}}^{n+k} P_{i}, j=1,2, \cdots, n ; Z_{n+q}=\bigcup_{\substack{i=0, i \neq q, n+q}}^{n+k} P_{i}, \quad q=1,2, \cdots, k
$$

Here the elements $P_{i}(i=1,2, \cdots, n+k)$ are bases sources, the element $P_{0}$ are sources of completeness of the semilattice $D$. Therefore $|X| \geq n+k$ (by symbol $|X|$ we denoted the power of a set $X)$, since $\left|P_{i}\right| \geq 1(i=1,2, \cdots, n+k)$ (see [1] [2] chapter 11).

In this paper we are learning irreducible generating sets of the semigroup $B_{X}(D)$ defined by semilattices of the class $\Sigma_{8}(X, n+k+1)$.

Note, that it is well known, when $k=2$, then generated sets of the complete semigroup of binary relations defined by semilattices of the class $\Sigma_{8}(X, 2+2+1)=\Sigma_{8}(X, 5)$.

In this paper we suppose, that $3 \leq k \leq n$.

Remark, that in this case (i.e. $k \geq 3$ ), from the formal equalities of a semilattice $D$ follows, that the intersections of any two elements of a semilattice $D$ is not empty.

Lemma 2.0 If $D \in \Sigma_{8}(X, n+k+1)$, then the following statements are true:

a) $\bigcap_{i=1}^{n+k} Z_{i}=P_{0}$

b) $Z_{j+1} \backslash Z_{j}=P_{j}, j=1,2, \cdots, n-1$;

c) $Z_{q} \backslash Z_{n+q}=P_{n+q}, q=1, \cdots, k$.

Proof. From the formal equalities of the semilattise $D$ immediately follows the following statements:

$$
\begin{aligned}
& \bigcap_{i=1}^{n+k} Z_{i}=P_{0}, \quad Z_{j+1} \backslash Z_{j}=\left(\bigcup_{\substack{i=0, i \neq j+1}}^{n+k} P_{i}\right) \backslash\left(\bigcup_{\substack{i=0, i \neq j}}^{n+k} P_{i}\right)=P_{j}, j=1,2, \cdots, n-1 ; \\
& Z_{q} \backslash Z_{n+q}=\left(\bigcup_{\substack{i=0, i \neq q}}^{n+k} P_{i}\right) \backslash\left(\bigcup_{\substack{i=0, i \neq q, n+q}}^{n+k} P_{i}\right)=P_{n+q}, \quad q=1, \cdots, k .
\end{aligned}
$$

The statements a), b) and c) of the lemma 2.0 are proved. 
Lemma 2.0 is proved.

We denoted the following sets by symbols $D_{1}, D_{2}$ and $D_{3}$ :

$$
D_{1}=\left\{Z_{1}, Z_{2}, \cdots, Z_{k}\right\}, D_{2}=\left\{Z_{k+1}, Z_{k+2}, \cdots, Z_{n}\right\}, D_{3}=\left\{Z_{n+1}, Z_{n+2}, \cdots, Z_{n+k}\right\} \text {. }
$$

Lemma 2.1. Let $D \in \Sigma_{8.0}(X, n+k+1)$ and $\alpha \in B_{X}(D)$. Then the following statements are true:

1) Let $T, T^{\prime} \in D_{2} \cup D_{3}, T \neq T^{\prime}$. If $T, T^{\prime} \in V(D, \alpha)$, then $\alpha$ is external element of the semigroup $B_{X}(D)$;

2) Let $T \in D_{1}, T^{\prime} \in D_{2} \cup D_{3}$. If $T^{\prime} \not \subset T$ and $T, T^{\prime} \in V(D, \alpha)$, then $\alpha$ is external element of the semigroup $B_{X}(D)$.

3) Let $T, T^{\prime} \in D_{1}$ and $T \neq T^{\prime}$. If $T, T^{\prime} \in V(D, \alpha)$ and $k \geq 3$, then $\alpha$ is external element of the semigroup $B_{X}(D)$;

Proof. Let $Z_{0}=\breve{D}$ and $\alpha=\delta \circ \beta$ for some $\delta, \beta \in B_{X}(D) \backslash\{\alpha\}$. If quasinormal representation of binary relation $\delta$ has a form

$$
\delta=\bigcup_{T \in V(D, \delta)}\left(Y_{T}^{\delta} \times T\right)
$$

then

$$
\alpha=\delta \circ \beta=\bigcup_{T \in V(D, \delta)}\left(Y_{T}^{\delta} \times T \beta\right) .
$$

From the formal equalities (2.0) of the semilattice $D$ we obtain that:

$$
\begin{aligned}
& Z_{0} \beta=\bigcup_{i=0}^{n+k} P_{i} \beta ; \quad Z_{j} \beta=\bigcup_{\substack{i=0, i \neq j}}^{n+k} P_{i} \beta, j=1,2, \cdots, n ; \\
& Z_{n+q} \beta=\bigcup_{\substack{i=0, i \neq q, n+q}}^{n+k} P_{i} \beta, \quad q=1,2, \cdots, k .
\end{aligned}
$$

where $P_{i} \beta \neq \varnothing \quad$ for any $P_{i} \neq \varnothing \quad(i=0,1,2, \cdots, n+k)$ and $\beta \in B_{X}(D)$ by definition of a semilattice $D$ from the class $\Sigma_{8.0}(X, n+k+1)$.

Now, let $Z_{m} \beta=T$ and $Z_{j} \beta=T^{\prime}$ for some $T \neq T^{\prime}, T, T^{\prime} \in D_{2} \cup D_{3}$, then from the equalities (2.3) follows that $T=P_{0} \beta=T^{\prime}$ since $T$ and $T^{\prime}$ are minimal elements of the semilattice $D$ and $P_{0} \neq \varnothing$ by preposition. The equality $T=T^{\prime}$ contradicts the inequality $T \neq T^{\prime}$.

The statement a) of the Lemma 2.1 is proved.

Now, let $Z_{m} \beta=T$ and $Z_{j} \beta=T^{\prime}$, for some $T \in D_{1}, T^{\prime} \in D_{2} \cup D_{3}$ and $T^{\prime} \not \subset T$, then from the equalities 2.3 follows, that

$$
\begin{aligned}
T^{\prime}=Z_{j} \beta=Z_{0} \beta=\bigcup_{i=0}^{n+k} P_{i} \beta, \text { if } j=0, \text { or } T^{\prime}=Z_{j} \beta=\bigcup_{\substack{i=0, i \neq j}}^{n+k} P_{i} \beta, 1 \leq j \leq n, \text { or } \\
T^{\prime}=Z_{n+q} \beta=\bigcup_{\substack{i=0, i \neq q, n+q}}^{n+k} P_{i} \beta
\end{aligned}
$$

where $j=n+q$. For the $Z_{j} \beta=T^{\prime}$ we consider the following cases:

1) If $T^{\prime}=Z_{0} \beta=\bigcup_{i=0}^{n+k} P_{i} \beta$, then we have

$$
P_{0} \beta=P_{1} \beta=\cdots=P_{n+k} \beta=T^{\prime},
$$

since $T^{\prime}$ is a minimal element of a semilattice $D$. On the other hand, 


$$
T=Z_{m} \beta=\left\{\begin{array}{l}
\bigcup_{\substack{i=0, i \neq m}}^{n+k} P_{i} \beta=\bigcup_{\substack{i=0, i \neq m}}^{n+k} T^{\prime}=T^{\prime}, \text { if } 1 \leq m \leq n ; \\
\bigcup_{\substack{i=0, i \neq q, n+q}}^{n+k} P_{i} \beta=\bigcup_{\substack{i=0, i \neq q, n+q}}^{n+k} T^{\prime}=T^{\prime}, \text { if } m=n+q .
\end{array}\right.
$$

But the equality $T=T^{\prime}$ contradicts the inequality $T \neq T^{\prime}$. Thus we have, that $j \neq 0$.

2) Let $1 \leq j \leq n$, i.e. $T^{\prime}=Z_{j} \beta=\bigcup_{\substack{i=0, i \neq j}}^{n+k} P_{i} \beta$, then we have, that

$$
P_{0} \beta=P_{1} \beta=\cdots=P_{j-1} \beta=P_{j+1} \beta=\cdots=P_{n+k} \beta=T^{\prime},
$$

since $T^{\prime}$ is a minimal element of a semilattice $D$. On the other hand:

$$
T=Z_{m} \beta=\left\{\begin{array}{l}
\left(\bigcup_{i=0}^{n+k} P_{i} \beta\right)=\left(\bigcup_{\substack{i=0, j \\
i \neq j}}^{n+k} P_{i} \beta\right) \cup P_{j} \beta=T^{\prime} \cup P_{j} \beta \text {, if } m=0 ; \\
\left(\bigcup_{\substack{i=0, i \neq m}}^{n+k} P_{i} \beta\right)=\left(\bigcup_{\substack{i=0, j \\
i \neq k}}^{n+k} P_{i} \beta\right) \cup P_{j} \beta=T^{\prime} \cup P_{j} \beta \text {, if } 1 \leq m \leq n, m \neq j ; \\
\left(\bigcup_{\substack{i=0, i \neq j, n+j}}^{n+k} P_{i} \beta\right)=T^{\prime}, \text { if } m=n+j ; \\
\left(\bigcup_{\substack{i=0, i \neq q, n+q}}^{n+k} P_{i} \beta\right)=\left(\bigcup_{\substack{i=0, i \neq q, n+q, j}}^{n+k} P_{i} \beta\right) \cup P_{j} \beta=T^{\prime} \cup P_{j} \beta, \text { if } m=n+q, q \neq j .
\end{array}\right.
$$

The equality $T=T^{\prime}$ contradicts the inequality $T \neq T^{\prime}$. Also, the equality $T=T^{\prime} \cup P_{j} \beta \quad\left(P_{j} \beta \in D\right) \quad$ contradicts the inequality $T \neq T^{\prime} \cup Z$ for any $Z \in D$ and $T^{\prime} \not \subset T$ ( $T^{\prime} \not \subset T$, by preposition) by definition of a semilattice $D$.

3) If $j=n+q \quad(1 \leq q \leq k)$, i.e. $T^{\prime}=Z_{n+q} \beta=\bigcup_{\substack{i=0, i \neq q, n+q}}^{n+k} P_{i} \beta$, then we have, that

$$
P_{0} \beta=P_{1} \beta=\cdots=P_{q-1} \beta=P_{q+1} \beta=\cdots=P_{n+q-1} \beta=P_{n+q+1} \beta=\cdots=P_{n+k} \beta=T^{\prime},
$$

since $T^{\prime}$ is a minimal element of a semilattice $D$. On the other hand:

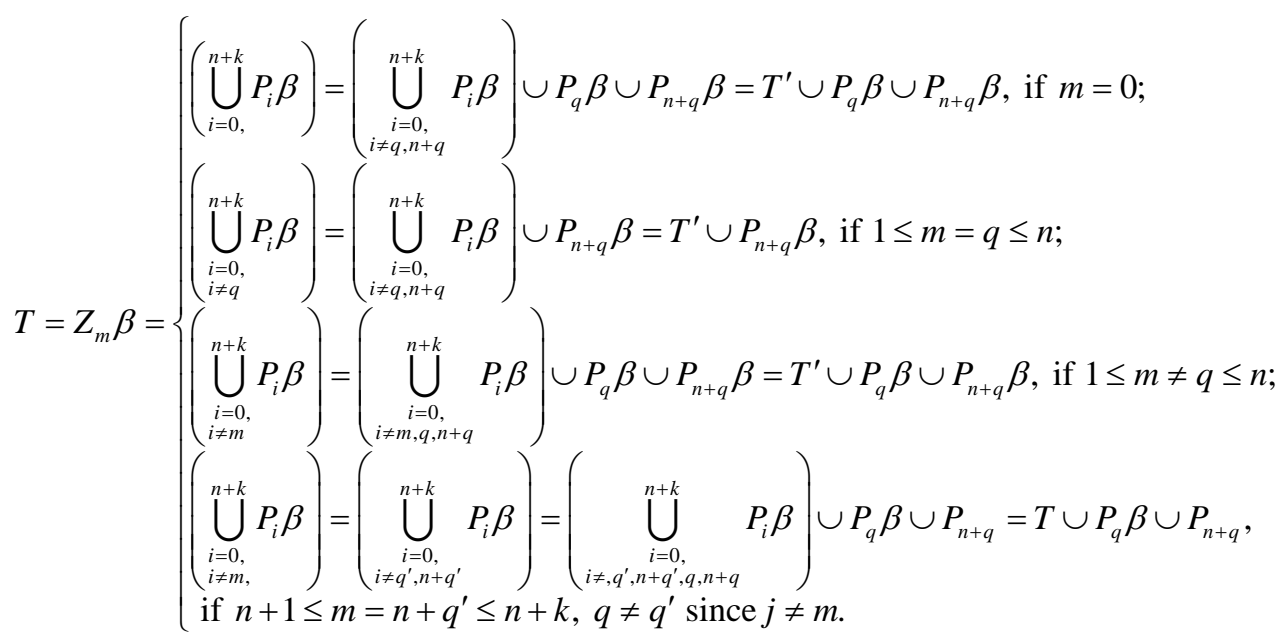


The equality $T=T^{\prime}$ contradicts the inequality $T \neq T^{\prime}$. Also, the equality $T=T^{\prime} \cup P_{q} \beta \cup P_{n+q} \beta$, or $T=T^{\prime} \cup P_{n+q} \beta \quad\left(P_{q} \beta, P_{n+q} \beta \in D\right)$ contradicts the inequality $T \neq T^{\prime} \cup Z$ for any $Z \in D$ and $T^{\prime} \not \subset T$ by definition of a semilattice $D$.

The statement 2) of the Lemma 2.1 is proved.

Let $T, T^{\prime} \in D_{1}$ and $T \neq T^{\prime}$. If $k \geq 3$ and $Z_{j} \beta=T^{\prime}, Z_{m} \beta=T$, then from the formal equalities (2.0) of a semilattice $D$ there exists such an element, that $P_{q} \subseteq Z_{j}$ and $P_{q} \subseteq Z_{m}$, where $0 \leq q \leq m+k$. So, from the equalities (2.3) follows that $P_{q} \beta \subseteq Z_{j} \beta=T^{\prime}$ and $P_{q} \beta \subseteq Z_{m} \beta=T$. Of from this and from the equalities (2.3) we obtain that there exists such an element $Z \in D$, for which the equalities $T^{\prime}=Z \cup Z^{\prime}$ and $T=Z \cup Z^{\prime \prime}$, where $Z^{\prime}, Z^{\prime \prime} \in D$. But such elements by definition of a semilattice $D$ do not exist.

The statement c) of the Lemma 2.1 is proved.

Lemma 2.1 is proved.

Lemma 2.2. Let $D \in \Sigma_{8}(X, n+k+1)$ and $\alpha \in B_{X}(D)$. Then the following statements are true:

1) Let $V(D, \alpha) \cap D_{1} \neq \varnothing, V(D, \alpha) \cap D_{2}=\varnothing, V(D, \alpha) \cap D_{3}=\varnothing$. If $\left|V(D, \alpha) \cap D_{1}\right| \geq 2$, then $\alpha$ is external element of the semigroup $B_{X}(D)$;

2) Let $V(D, \alpha) \cap D_{1}=\varnothing, V(D, \alpha) \cap D_{2} \neq \varnothing, V(D, \alpha) \cap D_{3}=\varnothing$. If $\left|V(D, \alpha) \cap D_{2}\right| \geq 2$, then $\alpha$ is external element of the semigroup $B_{X}(D)$;

3) Let $V(D, \alpha) \cap D_{1}=\varnothing, V(D, \alpha) \cap D_{2}=\varnothing, V(D, \alpha) \cap D_{3} \neq \varnothing$. If $\left|V(D, \alpha) \cap D_{3}\right| \geq 2$, then $\alpha$ is external element of the semigroup $B_{X}(D)$;

4) Let $V(D, \alpha) \cap D_{1}=\varnothing, V(D, \alpha) \cap D_{2} \neq \varnothing, V(D, \alpha) \cap D_{3} \neq \varnothing$, then $\alpha$ is external element of the semigroup $B_{X}(D)$;

5) Let $V(D, \alpha) \cap D_{1} \neq \varnothing, V(D, \alpha) \cap D_{2}=\varnothing, V(D, \alpha) \cap D_{3} \neq \varnothing$. If

$\left|V(D, \alpha) \cap D_{1}\right| \geq 2,\left|V(D, \alpha) \cap D_{3}\right|=1$, or $\left|V(D, \alpha) \cap D_{1}\right|=1$,

$\left|V(D, \alpha) \cap D_{3}\right| \geq 2$ then $\alpha$ is external element of the semigroup $B_{X}(D)$;

6) Let $V(D, \alpha) \cap D_{1} \neq \varnothing, V(D, \alpha) \cap D_{2} \neq \varnothing, V(D, \alpha) \cap D_{3}=\varnothing$, then $\alpha$ is external element of the semigroup $B_{X}(D)$;

7) Let $V(D, \alpha) \cap D_{1} \neq \varnothing, V(D, \alpha) \cap D_{2} \neq \varnothing, V(D, \alpha) \cap D_{3} \neq \varnothing$, then $\alpha$ is external element of the semigroup $B_{X}(D)$.

Proof. Let $\alpha$ be any element of the semigroup $B_{X}(D)$. It is easy that $V(D, \alpha) \in D$. We consider the following cases:

Let $V(D, \alpha) \cap D_{1}=\varnothing, V(D, \alpha) \cap D_{2}=\varnothing, V(D, \alpha) \cap D_{3}=\varnothing$, then $V(D, \alpha) \in\{\breve{D}\}$ since $V(D, \alpha)$ is subsemilattice of the semilattice $D$.

1) Let $V(D, \alpha) \cap D_{1} \neq \varnothing, V(D, \alpha) \cap D_{2}=\varnothing, V(D, \alpha) \cap D_{3}=\varnothing$.

If $\left|V(D, \alpha) \cap D_{1}\right|=1$, then $V(D, \alpha) \in\left\{Z_{j}\right\}$, or $V(D, \alpha) \in\left\{Z_{j}, \breve{D}\right\}$, where $j=1,2, \cdots, k$, since $V(D, \alpha)$ is subsemilattice of the semilattice $D$.

If $\left|V(D, \alpha) \cap D_{1}\right| \geq 2$, then by statement c) of the Lemma 2.1 follows that $\alpha$ is external element of the semigroup $B_{X}(D)$.

2) Let $V(D, \alpha) \cap D_{1}=\varnothing, V(D, \alpha) \cap D_{2} \neq \varnothing, V(D, \alpha) \cap D_{3}=\varnothing$.

If $\left|V(D, \alpha) \cap D_{2}\right|=1$, then $V(D, \alpha) \in\left\{Z_{j}\right\}$, or $V(D, \alpha) \in\left\{Z_{j}, \breve{D}\right\}$, where $j=k+1, k+2, \cdots, n$, since $V(D, \alpha)$ is a subsemilattice of the semilattice $D$. 
If $\left|V(D, \alpha) \cap D_{2}\right| \geq 2$, then by statement a) of the Lemma 2.1 follows that $\alpha$ is external element of the semigroup $B_{X}(D)$.

3) Let $V(D, \alpha) \cap D_{1}=\varnothing, V(D, \alpha) \cap D_{2}=\varnothing, V(D, \alpha) \cap D_{3} \neq \varnothing$.

If $\left|V(D, \alpha) \cap D_{3}\right|=1$, then $V(D, \alpha) \in\left\{Z_{j}\right\}$, or $V(D, \alpha) \in\left\{Z_{j}, \breve{D}\right\}$, $j=n+1, n+2, \cdots, n+k$, since $V(D, \alpha)$ is subsemilattice of the semilattice $D$.

If $\left|V(D, \alpha) \cap D_{3}\right| \geq 2$, then by statement a) of the Lemma 2.1 follows that $\alpha$ is external element of the semigroup $B_{X}(D)$.

4) Let $V(D, \alpha) \cap D_{1}=\varnothing, V(D, \alpha) \cap D_{2} \neq \varnothing, V(D, \alpha) \cap D_{3} \neq \varnothing$, then by the statement a) of the Lemma 2.1 follows that $\alpha$ is external element of the semigroup $B_{X}(D)$.

5) Let $V(D, \alpha) \cap D_{1} \neq \varnothing, V(D, \alpha) \cap D_{2}=\varnothing, V(D, \alpha) \cap D_{3} \neq \varnothing$.

If $\left|V(D, \alpha) \cap D_{1}\right|=1,\left|V(D, \alpha) \cap D_{3}\right|=1$, then $V(D, \alpha)=\left\{Z_{n+q}, Z_{q}\right\}$, or $V(D, \alpha)=\left\{Z_{n+q}, Z_{q}, \breve{D}\right\}$, or $V(D, \alpha)=\left\{Z_{n+q}, Z_{j}, \breve{D}\right\}$ where $Z_{1} \leq Z_{j} \leq Z_{k}$ and $q=1,2, \cdots, k$.

If $V(D, \alpha)=\left\{Z_{n+q}, Z_{j}, \breve{D}\right\}$ where $j \neq q, q=1,2, \cdots, k$, then by the statement 2) of the Lemma 2.1 follows that $\alpha$ is external element of the semigroup $B_{X}(D)$;

If $\left|V(D, \alpha) \cap D_{1}\right|=1,\left|V(D, \alpha) \cap D_{3}\right| \geq 2$, or $\left|V(D, \alpha) \cap D_{1}\right| \geq 2,\left|V(D, \alpha) \cap D_{3}\right|=1$, then from the statement 1) and 3) of the Lemma 2.1 follows that $\alpha$ is external element of the semigroup $B_{X}(D)$ respectively.

6) Let $V(D, \alpha) \cap D_{1} \neq \varnothing, V(D, \alpha) \cap D_{2} \neq \varnothing, V(D, \alpha) \cap D_{3}=\varnothing$. Then from the statement b) of the Lemma 2.1 follows that $\alpha$ is external element of the semigroup $B_{X}(D)$.

7) Let $V(D, \alpha) \cap D_{1} \neq \varnothing, V(D, \alpha) \cap D_{2} \neq \varnothing, V(D, \alpha) \cap D_{3} \neq \varnothing$, then by the statement a) of the Lemma 2.1 follows that $\alpha$ is external element of the semigroup $B_{X}(D)$.

Lemma 2.2 is proved.

Now we learn the following subsemilattices of the semilattice $D$ :

$$
\begin{aligned}
& \mathfrak{A}_{1}=\left\{\left\{Z_{n+j}, Z_{j}, \breve{D}\right\}\right\}, \text { where } j=1,2, \cdots, k ; \\
& \mathfrak{A}_{2}=\left\{\left\{Z_{j}, \breve{D}\right\}\right\}, \text { where } j=1,2, \cdots, n+k ; \\
& \mathfrak{A}_{3}=\left\{\left\{Z_{n+j}, Z_{j}\right\}\right\}, \text { where } j=1,2, \cdots, k ; \\
& \mathfrak{A}_{4}=\left\{\left\{Z_{j}\right\},\{\breve{D}\}\right\} \text {, where } j=1,2, \cdots, n+k .
\end{aligned}
$$

We denoted the following sets by symbols $\mathfrak{A}_{0}$ and $B\left(\mathfrak{A}_{0}\right)$ :

$$
\begin{aligned}
& \mathfrak{A}_{0}=\left\{V(D, \alpha) \subseteq D \mid V(D, \alpha) \notin \mathfrak{A}_{1} \cup \mathfrak{A}_{2} \cup \mathfrak{A}_{3} \cup \mathfrak{A}_{4}\right\}, \\
& B\left(\mathfrak{A}_{0}\right)=\left\{\alpha \in B_{X}(D) \mid V(D, \alpha) \in \mathfrak{A}_{0}\right\} .
\end{aligned}
$$

By definition of a set $B\left(\mathfrak{A}_{0}\right)$ follows that any element of the set is external element of the semigroup $B_{X}(D)$.

Lemma 2.3. Let $D \in \Sigma_{8}(X, n+k+1)$. If quasinormal representation of a binary relation $\alpha$ has a form 


$$
\alpha=\left(Y_{n+j}^{\alpha} \times Z_{n+j}\right) \cup\left(Y_{j}^{\alpha} \times Z_{j}\right) \cup\left(Y_{0}^{\alpha} \times \breve{D}\right),
$$

where $Y_{n+j}^{\alpha}, Y_{j}^{\alpha}, Y_{0}^{\alpha} \notin\{\varnothing\}$ and $j=1,2, \cdots, k$, then $\alpha$ is generated by elements of the elements of set $B\left(\mathfrak{A}_{0}\right)$.

Proof. 1). Let quasinormal representation of binary relations $\delta$ and $\beta$ have a form

$$
\begin{aligned}
& \delta=\left(Y_{n+j}^{\delta} \times Z_{n+j}\right) \cup\left(Y_{j}^{\delta} \times Z_{j}\right) \cup\left(Y_{q}^{\delta} \times Z_{q}\right) \cup\left(Y_{0}^{\delta} \times \breve{D}\right), \\
& \beta=\left(Z_{n+j} \times Z_{n+j}\right) \cup\left(\left(Z_{j} \backslash Z_{n+j}\right) \times Z_{j}\right) \cup\left(\left(\breve{D} \backslash Z_{j}\right) \times Z_{q}\right) \cup((X \backslash \breve{D}) \times \breve{D}),
\end{aligned}
$$

where $Y_{n+j}^{\alpha}, Y_{j}^{\alpha}, Y_{q}^{\alpha} \notin\{\varnothing\}, Z_{1} \leq Z_{q} \leq Z_{k}, q \neq j, j=1, \cdots, k$.

$$
\begin{aligned}
& Z_{n+j} \cup\left(Z_{j} \backslash Z_{n+j}\right) \cup\left(\breve{D} \backslash Z_{j}\right) \cup(X \backslash \breve{D}) \\
& =\left(P_{0} \cup \bigcup_{\substack{i=1, i \neq j, n+j}}^{n+k} P_{i}\right) \cup P_{n+j} \cup P_{j} \cup(X \backslash \breve{D})=\breve{D} \cup(X \backslash \breve{D})=X
\end{aligned}
$$

since the representation of a binary relation $\beta$ is quasinormal and by statement 3) of the Lemma 2.1 binary relations $\delta$ and $\beta$ are external elements of the semigroup $B_{X}(D)$. It is easy to see, that:

$$
\begin{gathered}
Z_{n+j} \beta=Z_{n+j}, \\
Z_{j} \beta=\left(P_{0} \cup \bigcup_{\substack{i=1, i \neq j}}^{n+k} P_{i}\right) \beta=\left(\left(P_{0} \cup \bigcup_{\substack{i=1, j \\
i \neq j, n+j}}^{n+k} P_{i}\right) \cup P_{n+j}\right) \beta \\
=Z_{n+j} \beta \cup P_{n+j} \beta=Z_{n+j} \cup Z_{j}=Z_{j}, \\
Z_{q} \beta=\left(P_{0} \cup \bigcup_{\substack{i=1, i \neq q}}^{n+k} P_{i}\right) \beta=Z_{n+j} \cup Z_{j} \cup Z_{q}=\breve{D}, \\
\breve{D} \beta=\bigcup_{i=0}^{n+k} P_{i} \beta=Z_{n+j} \cup Z_{j} \cup Z_{q}=\breve{D}
\end{gathered}
$$

since $\quad Z_{q} \cap Z_{n+j} \neq \varnothing, Z_{q} \cap\left(Z_{j} \backslash Z_{n+j}\right)=P_{n+j} \neq \varnothing, Z_{q} \cap\left(D \backslash Z_{j}\right)=P_{j} \neq \varnothing \quad$ (see equality (2.0))

$$
\begin{aligned}
\alpha & =\delta \circ \beta=\left(Y_{n+j}^{\delta} \times Z_{n+j} \beta\right) \cup\left(Y_{j}^{\delta} \times Z_{j} \beta\right) \cup\left(Y_{q}^{\delta} \times Z_{q} \beta\right) \cup\left(Y_{0}^{\delta} \times \breve{D} \beta\right) \\
& =\left(Y_{n+j}^{\delta} \times Z_{n+j}\right) \cup\left(Y_{j}^{\delta} \times Z_{j}\right) \cup\left(Y_{q}^{\delta} \times \breve{D}\right) \cup\left(Y_{0}^{\delta} \times \breve{D}\right) \\
& =\left(Y_{n+j}^{\delta} \times Z_{n+j}\right) \cup\left(Y_{j}^{\delta} \times Z_{j}\right) \cup\left(\left(Y_{q}^{\delta} \cup Y_{0}^{\delta}\right) \times \breve{D}\right)=\alpha,
\end{aligned}
$$

if $Y_{n+j}^{\delta}=Y_{n+j}^{\alpha}, \quad Y_{j}^{\delta}=Y_{j}^{\alpha}$ and $Y_{q}^{\delta} \cup Y_{0}^{\delta}=Y_{0}^{\alpha}$. Last equalities are possible since $\left|Y_{q}^{\delta} \cup Y_{0}^{\delta}\right| \geq 1 \quad\left(\left|Y_{0}^{\delta}\right| \geq 0\right.$, by preposition).

Lemma 2.3 is proved.

Lemma 2.4. Let $D \in \Sigma_{8}(X, n+k+1)$. If quasinormal representation of a binary relation $\alpha$ has a form $\alpha=\left(Y_{j}^{\alpha} \times Z_{j}\right) \cup\left(Y_{0}^{\alpha} \times \breve{D}\right)$, where $Y_{j}^{\alpha}, Y_{0}^{\alpha} \notin\{\varnothing\}$, $j=1,2, \cdots, n+k$, then binary relation $\alpha$ is generated by elements of the elements of set $B\left(\mathfrak{A}_{0}\right)$.

Proof. Let quasinormal representation of the binary relations $\delta$ and $\beta$ have a 
form:

$$
\begin{aligned}
& \delta=\left(Y_{j}^{\delta} \times Z_{j}\right) \cup\left(Y_{q}^{\delta} \times Z_{q}\right) \cup\left(Y_{0}^{\delta} \times \breve{D}\right), \\
& \beta=\left(Z_{j} \times Z_{j}\right) \cup\left(\left(\breve{D} \backslash Z_{j}\right) \times Z_{q}\right) \cup((X \backslash \breve{D}) \times \breve{D}),
\end{aligned}
$$

where $Y_{j}^{\delta}, Y_{q}^{\delta} \notin\{\varnothing\}$ and $Z_{1} \leq Z_{j} \neq Z_{q} \leq Z_{n+k}$. Then from the statements a), b) and c) of the Lemma 2.1 follows, that $\delta$ and $\beta$ are generated by elements of the set $B\left(\mathfrak{A}_{0}\right)$ and

$$
\begin{aligned}
& Z_{j} \beta=Z_{j}, \\
& Z_{q} \beta=\breve{D}, \text { since } Z_{q} \cap\left(D \backslash Z_{j}\right)=P_{j} \neq \varnothing, \\
& \breve{D} \beta=\breve{D} ; \\
& \delta \circ \beta=\left(Y_{j}^{\delta} \times Z_{j} \beta\right) \cup\left(Y_{q}^{\delta} \times Z_{q} \beta\right) \cup\left(Y_{0}^{\delta} \times \breve{D} \beta\right) \\
&=\left(Y_{j}^{\delta} \times Z_{j}\right) \cup\left(Y_{q}^{\delta} \times \breve{D}\right) \cup\left(Y_{0}^{\delta} \times \breve{D}\right) \\
&=\left(Y_{j}^{\delta} \times Z_{j}\right) \cup\left(\left(Y_{q}^{\delta} \cup Y_{0}^{\delta}\right) \times \breve{D}\right)=\alpha,
\end{aligned}
$$

if $Y_{j}^{\delta}=Y_{j}^{\alpha}, Y_{q}^{\delta} \cup Y_{0}^{\delta}=Y_{0}^{\alpha}$ and $q=1,2, \cdots, n+k$. Last equalities are possible since $\left|Y_{q}^{\delta} \cup Y_{0}^{\delta}\right| \geq 1 \quad\left(\left|Y_{0}^{\delta}\right| \geq 0\right.$ by preposition).

Lemma 2.4 is proved.

Lemma 2.5. Let $D \in \Sigma_{8}(X, n+k+1)$. If quasinormal representation of a binary relation $\alpha$ has a form $\alpha=\left(Y_{n+j}^{\alpha} \times Z_{n+j}\right) \cup\left(Y_{j}^{\alpha} \times Z_{j}\right)$, where $Y_{n+j}^{\alpha}, Y_{j}^{\alpha} \notin\{\varnothing\}$, $j=1,2, \cdots, k$, then binary relation $\alpha$ is generated by elements of the elements of set $B\left(\mathfrak{A}_{0}\right)$.

Proof. Let quasinormal representation of a binary relations $\delta, \beta$ have a form

$$
\begin{aligned}
& \delta=\left(Y_{n+j}^{\delta} \times Z_{n+j}\right) \cup\left(Y_{q}^{\delta} \times Z_{q}\right) \cup\left(Y_{0}^{\delta} \times \breve{D}\right), \\
& \beta=\left(Z_{n+j} \times Z_{n+j}\right) \cup\left(\left(\breve{D} \backslash Z_{n+j}\right) \times Z_{j}\right) \cup((X \backslash \breve{D}) \times \breve{D}),
\end{aligned}
$$

where $Y_{n+j}^{\delta}, Y_{q}^{\delta} \notin\{\varnothing\}, j \neq q$ and $j=1,2, \cdots, k$. Then from the Lemma 2.2 follows that $\beta$ is generated by elements of the set $B\left(\mathfrak{A}_{0}\right), \delta \in B\left(\mathfrak{A}_{0}\right)$ and

$$
Z_{n+j} \beta=Z_{n+j} \text {, }
$$

$$
Z_{q} \beta=Z_{n+j} \cup Z_{j}=Z_{j} \quad, \quad \text { since } \quad Z_{q} \cap Z_{n+j} \neq \varnothing \text {, }
$$

$Z_{q} \cap\left(\breve{D} \backslash Z_{n+j}\right)=P_{n+j} \neq \varnothing, j \neq q \quad$ (see equality (2.0))

$\breve{D} \beta=Z_{j}$ since $\breve{D} \cap(X \backslash \breve{D})=\varnothing$,

$$
\begin{aligned}
\delta \circ \beta & =\left(Y_{n+j}^{\delta} \times Z_{n+j} \beta\right) \cup\left(Y_{q}^{\delta} \times Z_{q} \beta\right) \cup\left(Y_{0}^{\delta} \times \breve{D}\right) \\
& =\left(Y_{n+j}^{\delta} \times Z_{n+j}\right) \cup\left(Y_{q}^{\delta} \times Z_{j}\right) \cup\left(Y_{0}^{\delta} \times Z_{j}\right) \\
& =\left(Y_{n+j}^{\delta} \times Z_{n+j}\right) \cup\left(\left(Y_{q}^{\delta} \cup Y_{0}^{\delta}\right) \times Z_{j}\right)=\alpha,
\end{aligned}
$$

if $Y_{n+j}^{\delta}=Y_{n+j}^{\alpha}$ and $Y_{q}^{\delta} \cup Y_{0}^{\delta}=Y_{j}^{\alpha}$. Last equalities are possible since $\left|Y_{q}^{\delta} \cup Y_{0}^{\delta}\right| \geq 1 \quad\left(\left|Y_{0}^{\delta}\right| \geq 0\right.$ by preposition).

Lemma 2.5 is proved.

Lemma 2.6. Let $D \in \Sigma_{8}(X, n+k+1)$. Then the following statements are true:

1) If quasinormal representation of a binary relation $\alpha$ has a form $\alpha=X \times Z_{j}$ 
$(j=1,2, \cdots, k)$, then binary relation $\alpha$ is generated by elements of the set $B\left(\mathfrak{A}_{0}\right)$.

2) If quasinormal representation of a binary relation $\alpha$ has a form $\alpha=X \times \breve{D}$, then binary relation $\alpha$ is generated by elements of the set $B\left(\mathfrak{A}_{0}\right)$.

Proof. 1) Let $T \in D \backslash\left(D_{2} \cup D_{3}\right)$. If quasinormal representation of a binary relations $\delta, \beta$ have a form

$$
\begin{aligned}
& \delta=\left(Y_{j}^{\delta} \times Z_{j}\right) \cup\left(Y_{0}^{\delta} \times \breve{D}\right), \\
& \beta=\left(Z_{n+j} \times Z_{n+j}\right) \cup\left(\left(Z_{j} \backslash Z_{n+j}\right) \times Z_{j}\right) \cup((X \backslash \breve{D}) \times \breve{D}),
\end{aligned}
$$

where $Y_{j}^{\delta}, Y_{0}^{\delta} \in\{\varnothing\}, \quad j=1,2, \cdots, k$

$$
\begin{aligned}
& Z_{n+j} \cup\left(Z_{j} \backslash Z_{n+j}\right) \cup(X \backslash \breve{D}) \\
& =\left(\bigcup_{\substack{i=0, i \neq j, n+j}}^{n+k} P_{i}\right) \cup\left(P_{j} \cup P_{n+j}\right) \cup(X \backslash \breve{D})=\breve{D} \cup(X \backslash \breve{D})=X
\end{aligned}
$$

(see equalities (2.0) and (2.1)), then from the Lemma 2.4 follows that $\delta$ is generated by elements of the set $B\left(\mathfrak{A}_{0}\right)$ and from the Lemma 2.3 element $\beta$ is generated by elements of the set $B\left(\mathfrak{A}_{0}\right)$ and

$$
\begin{gathered}
Z_{j} \beta=Z_{n+j} \cup Z_{j}=Z_{j}, \\
\breve{D} \beta=Z_{j}, \text { since } \breve{D} \cap(X \backslash \breve{D})=\varnothing, \\
\delta \circ \beta=\left(Y_{j}^{\delta} \times Z_{j} \beta\right) \cup\left(Y_{0}^{\delta} \times \breve{D} \beta\right)=\left(Y_{j}^{\delta} \times Z_{j}\right) \cup\left(Y_{0}^{\delta} \times Z_{j}\right)=X \times Z_{j}=\alpha,
\end{gathered}
$$

since representation of a binary relation $\delta$ is quasinormal.

The statement a) of the lemma 2.6 is proved.

2) Let quasinormal representation of a binary relation $\delta$ have a form

$$
\delta=\left(Z_{n+j} \times Z_{q}\right) \cup\left(\left(X \backslash Z_{n+j}\right) \times \breve{D}\right),
$$

where $j \neq q$, then from the Lemma 2.4 follows that $\delta$ is generated by elements of the set $B\left(\mathfrak{A}_{0}\right)$ and

$$
\begin{aligned}
Z_{q} \delta=\left(\bigcup_{\substack{i=0, i \neq q}}^{n+k} P_{i}\right) \delta & =\left(\bigcup_{\substack{i=0, i \neq q}}^{n+k} P_{i} \delta\right)=Z_{q} \cup \breve{D}=\breve{D}, \breve{D} \delta=\breve{D}, \text { since } \\
j \neq q, Z_{q} \delta \cap Z_{n+1} \neq \varnothing & \text { and } Z_{q} \delta \cap\left(X \backslash Z_{n+1}\right) \neq \varnothing ; \\
\delta \circ \delta & =\left(Z_{n+j} \times Z_{q} \delta\right) \cup\left(\left(X \backslash Z_{n+j}\right) \times \breve{D} \delta\right) \\
& =\left(Z_{n+j} \times \breve{D}\right) \cup\left(\left(X \backslash Z_{n+j}\right) \times \breve{D}\right)=X \times \breve{D}=\alpha
\end{aligned}
$$

since representation of a binary relation $\delta$ is quasinormal.

The statement $b$ ) of the lemma 2.6 is proved.

Lemma 2.6 is proved.

Lemma 2.7. Let $D \in \Sigma_{8}(X, n+k+1)$. Then the following statements are true:

a) If $|X \backslash \breve{D}| \geq 1$ and $T \in D_{2} \cup D_{3}$, then binary relation $\alpha=X \times T$ is generated by elements of the elements of set $B\left(\mathfrak{A}_{0}\right)$; 
b) If $X=\breve{D}$ and $T \in D_{2} \cup D_{3}$, then binary relation $\alpha=X \times T$ is external element for the semigroup $B_{X}(D)$.

Proof. 1) If quasinormal representation of a binary relation $\delta$ has a form

$$
\delta=\left(Y_{0}^{\delta} \times \breve{D}\right) \cup \bigcup_{j=k+1}^{n+k}\left(Y_{j}^{\delta} \times Z_{j}\right),
$$

where $Y_{j}^{\delta} \neq \varnothing$ for all $j=k+1, k+2, \cdots, n+k$, then $\delta \in B\left(\mathfrak{A}_{0}\right) \backslash\{\alpha\}$. Let quasinormal representation of a binary relations $\beta$ have a form $\beta=(\breve{D} \times T) \cup \bigcup_{t^{\prime} \in X \backslash \bar{D}}\left(\left\{t^{\prime}\right\} \times f\left(t^{\prime}\right)\right)$, where $f$ is any mapping of the set $X \backslash \breve{D}$ in the set $\left(D_{2} \cup D_{3}\right) \backslash\{T\}$. It is easy to see, that $\beta \neq \alpha$ and two elements of the set $D_{2} \cup D_{3}$ belong to the semilattice $V(D, \beta)$, i.e. $\delta \in B\left(\mathfrak{A}_{0}\right) \backslash\{\alpha\}$. In this case we have that $Z_{j} \beta=T$ for all $j=k+1, k+2, \cdots, n+k$.

$$
\begin{aligned}
\delta \circ \beta & =\delta=\left(Y_{0}^{\delta} \times \breve{D} \beta\right) \cup \bigcup_{j=k+1}^{n+k}\left(Y_{j}^{\delta} \times Z_{j} \beta\right) \\
& =\left(Y_{0}^{\delta} \times T\right) \cup \bigcup_{j=k+1}^{n+k}\left(Y_{j}^{\delta} \times T\right) \\
& =\left(\left(Y_{0}^{\delta} \cup \bigcup_{j=k+1}^{n+k} Y_{j}^{\delta}\right) \times T\right)=X \times T=\alpha,
\end{aligned}
$$

since the representation of a binary relation $\delta$ is quasinormal. Thus, the element $\alpha$ is generated by elements of the set $B\left(\mathfrak{A}_{0}\right)$.

The statement a) of the lemma 2.7 is proved.

2) Let $X=\breve{D}, \alpha=X \times T$, for some $T \in D_{2} \cup D_{3}$ and $\alpha=\delta \circ \beta$ for some $\delta, \beta \in B_{X}(D) \backslash\{\alpha\}$. Then we obtain that $Z_{j} \beta=T$ since $T$ is a minimal element of the semilattice $D$.

Now, let subquasinormal representations $\bar{\beta}$ of a binary relation $\beta$ have a form

$$
\bar{\beta}=\left(\left(\bigcup_{i=0}^{n+k} P_{i}\right) \times T\right) \cup \bigcup_{t^{\prime} \in X \backslash \bar{D}}\left(\left\{t^{\prime}\right\} \times \bar{\beta}_{2}\left(t^{\prime}\right)\right),
$$

where $\bar{\beta}_{1}=\left(\begin{array}{ccccc}P_{0} & P_{1} & P_{2} & \cdots & P_{n+k} \\ T & T & T & \cdots & T\end{array}\right)$ is normal mapping. But complement mapping $\bar{\beta}_{2}$ is empty, since $X \backslash \breve{D}=\varnothing$, i.e. in the given case, subquasinormal representation $\bar{\beta}$ of a binary relation $\beta$ is defined uniquely. So, we have that $\beta=\bar{\beta}=X \times T=\alpha \quad$ (see property 2 ) in the case 1.1), which contradict the condition, that $\beta \notin B_{X}(D) \backslash\{\alpha\}$.

Therefore, if $X=\breve{D}$ and $\alpha=X \times T$, for some $T \in D_{2} \cup D_{3}$, then $\alpha$ is external element of the semigroup $B_{X}(D)$.

The statement 2) of the Lemma 2.7 is proved.

Lemma 2.7 is proved.

Theorem 2.1. Let $D \in \Sigma_{8}(X, n+k+1), k \geq 3$, and

$$
\begin{gathered}
D_{1}=\left\{Z_{1}, Z_{2}, \cdots, Z_{k}\right\}, D_{2}=\left\{Z_{k+1}, Z_{k+2}, \cdots, Z_{n}\right\}, D_{3}=\left\{Z_{n+1}, Z_{n+2}, \cdots, Z_{n+k}\right\} ; \\
\mathfrak{A}_{1}=\left\{\left\{Z_{n+q}, Z_{q}, \breve{D}\right\}\right\}, \text { where } q=1,2, \cdots, k ;
\end{gathered}
$$




$$
\begin{gathered}
\mathfrak{A}_{2}=\left\{\left\{Z_{j}, \breve{D}\right\}\right\} \text {, where } j=1,2, \cdots, n+k ; \\
\mathfrak{A}_{3}=\left\{\left\{Z_{n+j}, Z_{j}\right\}\right\} \text {, where } j=1,2, \cdots, k ; \\
\mathfrak{A}_{4}=\left\{\left\{Z_{j}\right\},\{\breve{D}\}\right\} \text {, where } j=1,2, \cdots, n+k ; \\
\mathfrak{A}_{0}=\left\{V(D, \alpha) \subset D \mid V(D, \alpha) \notin \mathfrak{A}_{1} \cup \mathfrak{A}_{2} \cup \mathfrak{A}_{3} \cup \mathfrak{A}_{4}\right\}, \\
B\left(\mathfrak{A}_{0}\right)=\left\{\alpha \in B_{X}(D) \mid V(D, \alpha) \in \mathfrak{A}_{0}\right\}, \\
B_{0}=\left\{X \times T \mid T \notin D_{2} \cup D_{3}\right\}
\end{gathered}
$$

Then the following statements are true:

1) If $|X \backslash \breve{D}| \geq 1$, then the $S_{0}=B\left(\mathfrak{A}_{\circ}\right)$ is irreducible generating set for the semigroup $B_{X}(D)$;

2) If $X=\breve{D}$, then the $S_{1}=B_{0} \cup B\left(\mathfrak{A}_{\circ}\right)$ is irreducible generating set for the semigroup $B_{X}(D)$.

Proof. Let $D \in \Sigma_{8}(X, n+k+1), \quad k \geq 3$ and $|X \backslash \breve{D}| \geq 1$. First, we proved that every element of the semigroup $B_{X}(D)$ is generated by elements of the set $S_{0}$. Indeed, let $\alpha$ be an arbitrary element of the semigroup $B_{X}(D)$. Then quasinormal representation of a binary relation $\alpha$ has a form

$$
\alpha=\left(Y_{0}^{\alpha} \times \breve{D}\right) \cup \bigcup_{i=1}^{n+k}\left(Y_{i}^{\alpha} \times Z_{i}\right),
$$

where $\bigcup_{i=0}^{n+k} Y_{i}^{\alpha}=X \quad$ and $Y_{i}^{\alpha} \cap Y_{j}^{\alpha}=\varnothing \quad(0 \leq i \neq j \leq n+k)$. For the $V\left(X^{*}, \alpha\right)$ we consider the following cases:

1) If $V\left(X^{*}, \alpha\right) \notin \mathfrak{A}_{1} \cup \mathfrak{A}_{2} \cup \mathfrak{A}_{3} \cup \mathfrak{A}_{4}$, then $\alpha \in B\left(\mathfrak{A}_{\circ}\right) \subseteq S_{0}$ by definition of a set $S_{0}$.

Now, let $V\left(X^{*}, \alpha\right) \in \mathfrak{A}_{1} \cup \mathfrak{A}_{2} \cup \mathfrak{A}_{3} \cup \mathfrak{A}_{4}$.

2) If $V\left(X^{*}, \alpha\right) \in \mathfrak{A}_{1}$, then quasinormal representation of a binary relation $\alpha$ has a form $\alpha=\left(Y_{n+j}^{\alpha} \times Z_{n+j}\right) \cup\left(Y_{j}^{\alpha} \times Z_{j}\right) \cup\left(Y_{0}^{\alpha} \times \breve{D}\right)$, where $Y_{n+j}^{\alpha}, Y_{j}^{\alpha}, Y_{0}^{\alpha} \notin\{\varnothing\}$ $(j=1,2, \cdots, k)$ and from the Lemma 2.3 follows that $\alpha$ is generated by elements of the elements of set $B\left(\mathfrak{A}_{0}\right) \subseteq S_{0}$ by definition of a set $S_{0}$.

3) If $V\left(X^{*}, \alpha\right) \in \mathfrak{A}_{2}$, then quasinormal representation of a binary relation $\alpha$ has a form $\alpha=\left(Y_{j}^{\alpha} \times Z_{j}\right) \cup\left(Y_{0}^{\alpha} \times \breve{D}\right)$, where $Y_{j}^{\alpha}, Y_{0}^{\alpha} \notin\{\varnothing\}, j=1,2, \cdots, n+k$ and from the Lemma 2.4 follows that $\alpha$ is generated by elements of the elements of set $B\left(\mathfrak{A}_{0}\right) \subseteq S_{0}$ by definition of a set $S_{0}$.

4) If $V\left(X^{*}, \alpha\right) \in \mathfrak{A}_{3}$, then quasinormal representation of a binary relation $\alpha$ has a form $\alpha=\left(Y_{n+j}^{\alpha} \times Z_{n+j}\right) \cup\left(Y_{j}^{\alpha} \times Z_{j}\right)$, where $Y_{n+j}^{\alpha}, Y_{j}^{\alpha} \notin\{\varnothing\}, j=1,2, \cdots, k$ and from the Lemma 2.5 follows that $\alpha$ is generated by elements of the elements of set $B\left(\mathfrak{A}_{0}\right) \subseteq S_{0}$ by definition of a set $S_{0}$.

Now, let $V\left(X^{*}, \alpha\right) \in \mathfrak{A}_{4}$, then quasinormal representation of a binary relation $\alpha$ has a form $\alpha=X \times \breve{D}$, or $\alpha=X \times Z_{j}$, where $j=1,2, \cdots, n+k$.

5) If $\alpha=X \times \breve{D}$, then from the statement b) of the Lemma 2.6 follows that binary relation $\alpha$ is generated by elements of the set $B\left(\mathfrak{A}_{0}\right)$. 
6) If $\alpha=X \times Z_{j}$, where $j=1,2, \cdots, n+k$, then from the statement a) of the Lemma 2.6 and 2.7 follows that binary relation $\alpha$ is generated by elements of the set $B\left(\mathfrak{A}_{0}\right)$.

Thus, we have that $S_{0}$ is a generating set for the semigroup $B_{X}(D)$. If $|X \backslash \breve{D}| \geq 1$, then the set $S_{0}$ is an irreducible generating set for the semigroup $B_{X}(D)$ since, $S_{0}$ is a set external elements of the semigroup $B_{X}(D)$.

The statement a) of the Theorem 2.1 is proved.

Now, let $X=\breve{D}$. First, we proved that every element of the semigroup $B_{X}(D)$ is generated by elements of the set $S_{1}$. The cases 1$\left.\left.\left.), 2\right), 3\right), 4\right)$ and 5) are proved analogously of the cases 1),2),3), 4) and 5 given above and consider case, when $V\left(X^{*}, \alpha\right) \in \mathfrak{A}_{1}$.

If $V\left(X^{*}, \alpha\right)=Z_{j}$, where $j=1,2, \cdots, k$, then from the statement a) of the Lemma 2.7 follows that binary relation $\alpha$ is generated by elements of the set $B\left(\mathfrak{A}_{0}\right)$.

If $V\left(X^{*}, \alpha\right)=Z_{j}$, where $Z_{j} \in D_{2} \cup D_{3}$, then from the statement $\mathrm{b}$ ) of the Lemma 2.6 follows that binary relation $\alpha=X \times T$ is external element for the semigroup $B_{X}(D)$.

Thus, we have that $S_{1}$ is a generating set for the semigroup $B_{X}(D)$.

If $X=\breve{D}$, then the set $S_{1}$ is an irreducible generating set for the semigroup $B_{X}(D)$ since $S_{1}$ is a set external elements of the semigroup $B_{X}(D)$.

The statement $\mathrm{b}$ ) of the Theorem 2.1 is proved.

Theorem 2.1 is proved.

Corollary 2.1. Let $D \in \Sigma_{8}(X, n+k+1) \quad(k \geq 3)$ and

$$
\begin{gathered}
D_{1}=\left\{Z_{1}, Z_{2}, \cdots, Z_{k}\right\}, D_{2}=\left\{Z_{k+1}, Z_{k+2}, \cdots, Z_{n}\right\}, D_{3}=\left\{Z_{n+1}, Z_{n+2}, \cdots, Z_{n+k}\right\} ; \\
\mathfrak{A}_{1}=\left\{\left\{Z_{n+q}, Z_{q}, \breve{D}\right\}\right\}, \text { where } q=1,2, \cdots, k ; \\
\mathfrak{A}_{2}=\left\{\left\{Z_{j}, \breve{D}\right\}\right\}, \text { where } j=1,2, \cdots, n+k ; \\
\mathfrak{A}_{3}=\left\{\left\{Z_{n+j}, Z_{j}\right\}\right\} \text {, where } j=1,2, \cdots, k ; \\
\mathfrak{A}_{4}=\left\{\left\{Z_{j}\right\},\{\breve{D}\}\right\}, \text { where } j=1,2, \cdots, n+k ; \\
\mathfrak{A}_{0}=\left\{V(D, \alpha) \subset D \mid V(D, \alpha) \notin \mathfrak{A}_{1} \cup \mathfrak{A}_{2} \cup \mathfrak{A}_{3} \cup \mathfrak{A}_{4}\right\}, \\
B\left(\mathfrak{A}_{0}\right)=\left\{\alpha \in B_{X}(D) \mid V(D, \alpha) \in \mathfrak{A}_{0}\right\}, \\
B_{0}=\left\{X \times T \mid T \notin D_{2} \cup D_{3}\right\}
\end{gathered}
$$

Then the following statements are true:

1) If $|X \backslash \breve{D}| \geq 1$, then $S_{0}=B\left(\mathfrak{A}_{\circ}\right)$ is the uniquely defined generating set for the semigroup $B_{X}(D)$;

2) If $X=\breve{D}$, then $S_{1}=B_{0} \cup B\left(\mathfrak{A}_{0}\right)$ is the uniquely defined generating set for the semigroup $B_{X}(D)$.

Proof. It is well known, that if $B$ is all external elements of the semigroup $B_{X}(D)$ and $B^{\prime}$ is any generated set for the $B_{X}(D)$, then $B \subseteq B^{\prime}$ (see [1] [2] Lemma 1.15.1). From this follows that the sets $S_{0}=B\left(\mathfrak{A}_{0}\right)$ and 
$S_{1}=B_{0} \cup B\left(\mathfrak{A}_{\circ}\right)$ are defined uniquely, since they are sets external elements of the semigroup $B_{X}(D)$.

Corollary 2.1 is proved.

It is well-known, that if $B$ is all external elements of the semigroup $B_{X}(D)$ and $B^{\prime}$ is any generated set for the $B_{X}(D)$, then $B \subseteq B^{\prime}$ (Definition 1.1).

In this article, we find irredusible generating set for the complete semigroups of binary relations defined by $X$-semilattices of unions of the class $\Sigma_{8}(X, n+k+1) \quad(k \geq 3)$. This generating set is uniquely defined, since they are defined by elements of the external elements of the semigroup $B_{X}(D)$.

\section{References}

[1] Diasamidze, Y. and Makharadze, S. (2013) Complete Semigroups of Binary Relations. Kriter, Turkey, 1-519.

[2] Я. И. Диасамидзе, Ш. И. Махарадзе (2017) Полные полугруппы бинарных отношений. Lambert Academic Publishing, 1-692. 